\title{
Identification of different trypanosome species in the mid-guts of tsetse flies of the Malanga (Kimpese) sleeping sickness focus of the Democratic Republic of Congo
}

Gustave Simo $^{1 *}$, Barberine Silatsa ${ }^{1}$, Njiokou Flobert ${ }^{2}$, Pascal Lutumba ${ }^{3}$, Philemon Mansinsa ${ }^{4}$, Joule Madinga ${ }^{3}$, Emile Manzambi ${ }^{5}$, Reginald De Deken ${ }^{6}$ and Tazoacha Asonganyi ${ }^{7}$

\begin{abstract}
Background: The Malanga sleeping sickness focus of the Democratic Republic of Congo has shown an epidemic evolution of disease during the last century. However, following case detection and treatment, the prevalence of the disease decreased considerably. No active survey has been undertaken in this focus for a couple of years. To understand the current epidemiological status of sleeping sickness as well as the animal African trypanosomiasis in the Malanga focus, we undertook the identification of tsetse blood meals as well as different trypanosome species in flies trapped in this focus.
\end{abstract}

Methods: Pyramidal traps were use to trap tsetse flies. All flies caught were identified and live flies were dissected and their mid-guts collected. Fly mid-gut was used for the molecular identification of the blood meal source, as well as for the presence of different trypanosome species.

Results: About 949 Glossina palpalis palpalis were trapped; 296 (31.2\%) of which were dissected, 60 (20.3\%) blood meals collected and 57 (19.3\%) trypanosome infections identified. The infection rates were 13.4\%, 5.1\%, 3.5\% and 0.4\% for Trypanosoma congolense savannah type, Trypanosoma brucei s.l., Trypanosoma congolense forest type and Trypanosoma vivax, respectively. Three mixed infections including Trypanosoma brucei s.l. and Trypanosoma congolense savannah type, and one mixed infection of Trypanosoma vivax and Trypanosoma congolense savannah type were identified. Eleven Trypanosoma brucei gambiense infections were identified; indicating an active circulation of this trypanosome subspecies. Of all the identified blood meals, about $58.3 \%$ were identified as being taken on pigs, while $33.3 \%$ and $8.3 \%$ were from man and other mammals, respectively.

Conclusion: The presence of Trypanosoma brucei in tsetse mid-guts associated with human blood meals is indicative of an active transmission of this parasite between tsetse and man. The considerable number of pig blood meals combined with the circulation of Trypanosoma brucei gambiense in this focus suggests a transmission cycle involving humans and domestic animals and could hamper eradication strategies. The various species of trypanosomes identified in the Malanga sleeping sickness focus indicates the coexistence of animal and human African Trypanosomiasis. The development of new strategies integrating control measures for human and animal trypanosomiasis may enable the reduction of the control costs in this locality.

Keywords: Sleeping sickness, Animal African trypanosomiasis, Trypanosomes, Blood meals, Tsetse flies, Mid-guts

\footnotetext{
* Correspondence: gsimoca@yahoo.fr

'Department of Biochemistry, Faculty of Science, University of Dschang, PO

Box 67, Dschang, Cameroon

Full list of author information is available at the end of the article
} 


\section{Background}

Sleeping sickness or Human African trypanosomiasis (HAT) is a fatal disease that occurs only in sub-Saharan Africa. About 60 million persons are exposed to the risk of infection with about 10,000 new cases reported per year [1]. The disease cycle includes three components: the trypanosome (Trypanosoma brucei gambiense or Trypanosoma brucei rhodesiense), the tsetse fly (Glossina), and the host (human or animal). HAT occurs generally in rural areas, and during the end of the $20^{\text {th }}$ century, many outbreaks in historical foci were observed [2]. Faced with these outbreaks, considerable efforts were deployed to fight against this disease. This resulted in a considerable decrease in transmission rate and a consequential decrease in surveillance.

The keystones of interventions against HAT are active and passive case finding, followed by treatment, vector control, and animal reservoir management. However, in most affected countries, case finding followed by treatment are the cornerstones of the disease control. This strategy was shown to permit a reduction in the number of reported new cases by about $69 \%$ during the period from 1997-2006 [3]. Following this success, the elimination of the disease was declared. To avoid the reemergence of the disease, as was the case in 1960; control and surveillance of the disease must not be abandoned. In fact, in 1960 when HAT was under control in most endemic countries, the advent of independence in most of the countries led to a decline in disease awareness, due to the rarity of HAT cases, and because the newly independent authorities had other priorities. This has resulted in the resurgence of the disease in most historical foci. This resurgence was probably due to the presence of undiagnosed patients during case detection, an increase in the population of infected flies, which can transmit the parasite to healthy people, and to the presence of animal reservoirs from which tsetse flies can get infected.

The current epidemiological context of HAT is similar to the situation 50 years ago. This suggests that if there is no improvement of surveillance strategies, there may be a decline in awareness of the disease, since most endemic countries are faced with new challenges like HIV/AIDS, malaria, tuberculosis. The consequence will be a slow return of the disease over time, and there will be a resurgence of the disease in most former endemic areas. To avoid such an eventuality, there is need to introduce new control and surveillance strategies involving new measures, since elimination of HAT as a public health problem requires continuous efforts and innovative approaches. The transmission cycle shows tsetse flies as the central actors that transmit the parasite to vertebrate hosts. Therefore, the integration of tsetse flies as an important component of new control or surveillance strategies is crucial if the disease is to be eliminated. For example, the identification of T. b. gambiense in the tsetse fly is an indication that the transmission of the parasite occurs in the disease focus. In addition, the identification of infected flies and the localization of areas where tsetse flies take most of their blood meals may enable areas of high disease transmission to be defined. In the past, such approaches were difficult to undertake due to difficulties in identifying T. b. gambiense in tsetse flies, and identifying various tsetse fly vertebrate hosts. During the last decades, molecular tools have been developed to identify blood meals $[4,5]$ and different trypanosome species in tsetse flies and mammals [6,7]. For instance, the identification of trypanosomes in mammals and vectors has been considerably improved by the PCR-based methods [6,8-12]. These tools provided data that enabled the understanding of the disease transmission cycle as well as the epidemiology of HAT.

In the present work, we aimed to identify the origin of blood meals and the trypanosome species present in the mid-guts of tsetse flies caught in the Malanga HAT focus of the Bas Congo Province of the Democratic Republic of Congo, in order to understand the current epidemiological situation of the disease and to generate data enabling an understanding of animal trypanosomosis in this locality.

\section{Methods}

\section{Study zone}

The Malanga $\left(4^{\circ} 34^{\prime} \mathrm{S}, 14^{\circ} 21^{\prime} \mathrm{E}\right)$ HAT focus is located in the Bas Congo Province of the Democratic Republic of Congo (Figure 1). This focus lies along the Lukunga River and has vegetation that is characterized by the presence of herbaceous savannah and forest relics. The Malanga focus is situated at $232 \mathrm{~km}$ from Kinshasa and $133 \mathrm{~km}$ from Matadi. Its altitude is estimated at 340 meters. The inhabitants of this area mostly practice subsistence farming and small scale animal husbandry. Farms situated along valleys and domestic animals such as goats, sheep and pigs are found in this focus, most of which move freely around the village. Glossina palpalis palpalis is the only tsetse fly species found in this focus. No vector control activities have been deployed in this area for several years. During the last decade, the Malanga HAT focus was among the most active foci in the Democratic Republic of Congo (DRC). However, during the last couple of years the epidemiological situation in this focus has not been examined.

\section{Entomologic survey}

The entomological survey was performed in August 2009 during the dry season. During this survey, 33 pyramidal traps [13] were deployed for 4 consecutive days in two capture sites ("Malanga gare" and Malanga village) of the Malanga HAT focus (Figure 2). For each trap, the geographical coordinates were recorded using a 


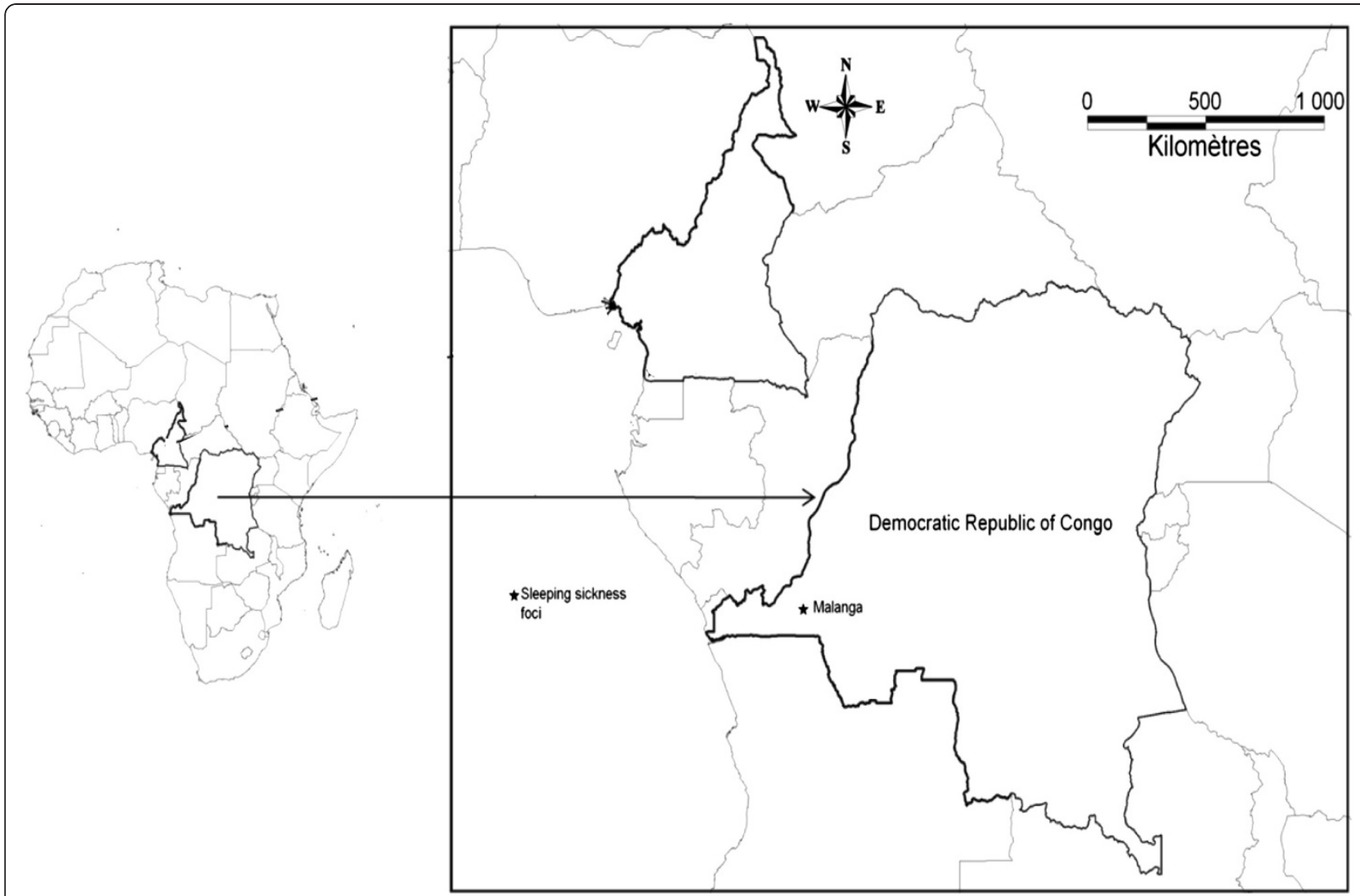

Figure 1 Map showing the geographic location of the Malanga sleeping sickness focus in the Democratic Republic of Congo.

Global Positioning System (GPS). Tsetse flies were collected twice a day, counted, and the species and sex of each of them determined. Living flies were dissected and the mid-gut extracted. In general, tsetse flies were dissected the same day. However, in instances where all flies could not be dissected on the same day of collection, they were wrapped up in wet floor cloth and dissected early the next morning before the arrival of the first round of collection. After dissection, the presence of residual blood meals in tsetse mid-gut was recorded. The mid-guts were collected into micro-tubes containing ethanol and maintained in the field at about $25^{\circ} \mathrm{C}$. In the laboratory, the tubes were kept at $-20{ }^{\circ} \mathrm{C}$ until the extraction of DNA.

\section{DNA extraction}

In the laboratory, the alcohol in the micro-tubes was evaporated for 60 minutes in an oven set at $80^{\circ} \mathrm{C}$. Subsequently, $300 \mu \mathrm{l}$ of Chelex 5\% was added to each tube and the mixture vortexed for 10 minutes. Thereafter, the tubes were incubated first at $56{ }^{\circ} \mathrm{C}$ for 30 minutes, and then at $98{ }^{\circ} \mathrm{C}$ for 60 minutes. The tubes were centrifuged at $14000 \mathrm{rpm}$ for 5 minutes and the supernatant (DNA extract) was collected and stored at $-20{ }^{\circ} \mathrm{C}$ until used.

\section{Identification of the blood meal origins}

Blood meals were identified using Heteroduplex PCR assay as described by Njiokou et al. [4]. Briefly, the amplification of cytochrome B gene was performed in $25 \mu \mathrm{l}$ of a mixture containing $5 \mu \mathrm{l}$ of DNA extract and 20 pmol of each vertebrate cytochrome B primers (Cyt B1/ 2) [5]. From the amplified DNA fragments, heteroduplexes were formed by hybridization with Giant rat cytochrome B DNA chosen as a driver [4]. The heteroduplex DNA profiles were resolved on $5 \%$ acrylamide/urea gel as described by Njiokou et al. [4]. The blood meal origin was identified by comparing the heteroduplex profiles of each blood meal with those of reference vertebrate hosts commonly found in this HAT focus like man and domestic animals (pig, goat, and sheep).

\section{Identification of different species of trypanosomes}

For this identification, two approaches were used: the first approach focused on the internal transcribed spacer 1 (ITS) of ribosomal DNA as described by Desquesnes et al. [14], whereas the second approach used primers specific to Trypanozoon [15], T. congolense forest type [15], T. congolense savannah type [15], T. vivax [15] and T. simiae [15]. 


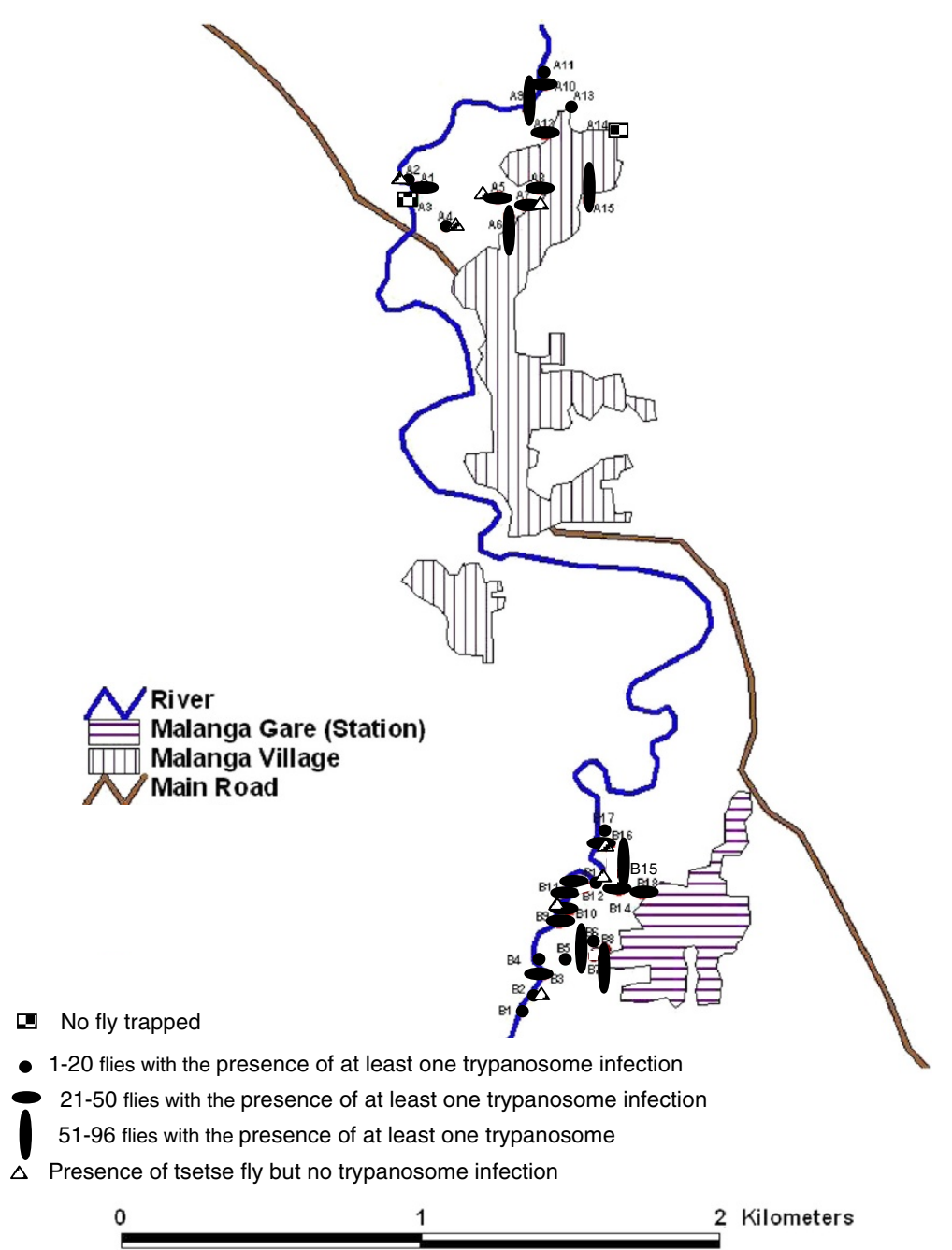

Figure 2 Capture sites presenting the localization of the traps with the range of trapped flies per trap as well as the traps where trypanosome infections were identified.

For the ITS, the amplification reactions were carried out as described by Farikou et al. [16]. Briefly, two pairs of primers designed for ribosomal DNA were used. The first amplification round was performed in a final volume of $25 \mu \mathrm{l}$ containing $2 \mu \mathrm{l}$ of DNA extract (template), 20 picomoles of each primer (TRYP18.2C: 5'-GCAAATTGCCCAATGTCG-3' TRYP4R: 5'-GCTGCGTTCTTCAACGA A-3'), $200 \mathrm{mM}$ of each dNTP and 0.5 unit of Taq DNA polymerase. One denaturing step at $94{ }^{\circ} \mathrm{C}$ for 3 minutes was followed by 30 amplification cycles. Each cycle consisted of a denaturation step at $94{ }^{\circ} \mathrm{C}$ for 30 seconds, an annealing step at $51{ }^{\circ} \mathrm{C}$ for 30 seconds, and an extension step at $72{ }^{\circ} \mathrm{C}$ for 2 minutes and a final extension at $72{ }^{\circ} \mathrm{C}$ for 10 minutes. The amplified products were diluted 10 fold and $2 \mu \mathrm{l}$ of each dilution was used as a template for the nested PCR. This latter was performed using primers IRFCC (5'CCTGCAGCTGGATCAT 3') and TRYP5RCG (5'ATCGCGACACGTTGTG 3'). The conditions and the amplification program for the nested PCR were identical to those described for the first PCR. Then micro-liters of the amplified products of the nested PCR were resolved on $2 \%$ agarose gel containing $10 \mathrm{mg} / \mu \mathrm{l}$ of ethidium bromide. The gels were observed on ultraviolet light and photographed.

For the second approach which uses primers specific to Trypanozoon [15], T. congolense forest type [15], T. congolense savannah type [15], T. vivax [15] and T. simiae [15], the amplification conditions were as described previously [17].

To identify $T . b$. gambiense, all samples with a multicopy $177 \mathrm{bp}$ repeat sequence specific for trypanosomes of the subgenus Trypanozoon or for which a DNA fragment of a molecular weight corresponding to that of $T$. brucei (Figure 3) were selected. From these samples, a second PCR was performed to search for the presence of T. b. gambiense. This second PCR was carried out as described by Simo et al. [17] using TRBPA1/2 primers 


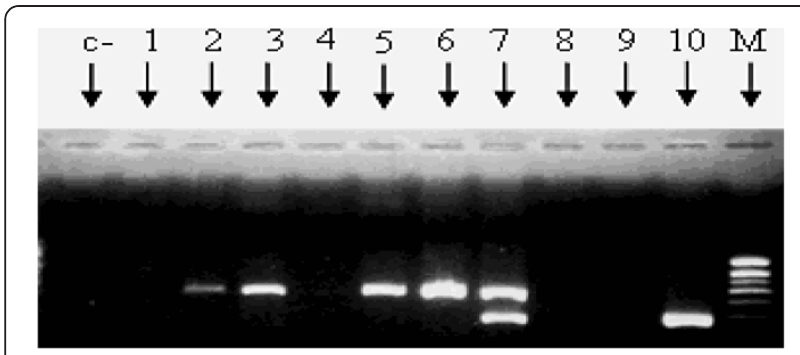

Figure 3 Example of agarose gel showing the resolution of the amplified products of ITS-1 of different trypanosome species. M: Molecular marker; C- : negative control; 1, 4, 8 and 9: samples showing no trypanosome infection; 2: T. congolense positive control; 10: T. brucei s.l. positive control; 5 and 6; samples showing $T$. congolense infections; 7 : samples showing mixed infections of $T$. brucei s.l. and T. congolense.

that amplify an allele of 149 bp characteristic of group 1 T. b. gambiense [18]. All samples that did not shown the allele of $149 \mathrm{bp}$ through the second PCR were considered here as being infected by $T$. b. brucei, since the other species (Trypanosoma evansi and Trypanosoma brucei rhodesiense) of the subgenus Trypanozoon are probably absent in the region due to their geographical localization. Since T. brucei s.l. is a diploid organism, a mixed infection of T. b. brucei and T. b. gambiense was identified when TRBPA1/2 primers showed more than two alleles.

\section{Results}

\section{Entomological survey}

During the entomological survey, 33 pyramidal traps were deployed and 949 Glossina palpalis palpalis were trapped (Table 1): 563 females and 386 males. Two hundred and ninety six tsetse flies (31.2\%) were dissected and 42 teneral flies (fly which had never taken a blood meal) identified. About $70 \%$ of trapped flies died in the trap or during the period between their capture and the dissection. Most traps deployed had at least one tsetse fly during the four days of capture. The range of tsetse flies caught per trap is reported in Figure 2. The average apparent density of tsetse flies per day was 7.2 for both sites and 7.3 for site A and 6.82 for site B (Table 1). Among the dissected flies, $60 \quad 6.33 \%$ of the overall trapped flies) blood meals were collected.

\section{Blood meal identification}

Of the 60 blood meals sampled, the origin of 55 (91.7\%) of them was successfully identified. In addition to using human, domestic animals (pig, goat, sheep) commonly found in this HAT focus, 15 wild animal species were also used as references [19]. Five (8.3\%) blood meals could not be identified because their heteroduplex profiles did not correspond to those of the vertebrate hosts used as references. Of the 55 blood meals successfully identified, 35 (58.3\%) were from pigs, 20 (33.3\%) from man and $5(8.3 \%)$ from other mammals not yet identified (Table 1).

\section{Identification of trypanosome species}

Out of 296 tsetse flies dissected, the identification of trypanosomes was performed on 254 mid-guts because 42 were teneral flies. Of the 254 mid-guts analyzed, the amplification of internal transcribed spacers and the specific primers to Trypanozoon, $T$. congolense forest and $T$. congolense savannah type enabled us to identify 57 (22.4\%) mid-guts infected by at least one trypanosome species (whatever the species). Among these 57 infections, $75.4 \%(43 / 57)$ were due to trypanosomes of the subgenus Nannomonas. No T. simiae infection was identified either by internal transcribed spacer or $T$. simiae specific primers. Looking at the distribution of trypanosomes per trap, it appears that most traps caught tsetse flies infected by at least one species of trypanosome (Figure 2).

Of the 57 infections revealed by both methods (ITS of rDNA and species specific PCR), the ITS revealed 48 (20.25\%) mid-guts infected by different trypanosome species or subspecies, including one Duttonella ( $T$. vivax), 4 Trypanozoon and 43 Nannomonas (T. congolense forest and savannah types). The 43 Nannomonas infections included 9 (21\%) T. congolense forest type and 34 (79\%) T. congolense savannah type. Four mixed infections involving different trypanosome species were identified (Figure 3): three mixed infections of Trypanozoon and $T$. congolense savannah type, and one mixed infection of $T$. vivax and $T$. congolense savannah type.

The use of Trypanozoon specific primers, which target a multi-copy 177 bp repeat sequence revealed 13 (5.1\%) mid-guts infected with T. brucei s.l. The four T. brucei mid-gut infections identified by ITS of rDNA method were confirmed by the Trypanozoon specific primers. Moreover, nine additional T. brucei mid-gut infections were identified by the second method. The number of $T$. brucei infections revealed by the Trypanozoon specific primers was more than 3 times the number of infections revealed by ITS.

The TRBPA primers [18] that amplified an allele of 149 bp characteristic of group 1T. b. gambiense, revealed 11 (4.3\%: 11/254) mid-guts infected with this trypanosome subspecies among the 13 Trypanozoon positive samples. Moreover, 6 mixed infections involving T. b. gambiense and T. b. brucei were identified.

Of the 254 tsetse flies dissected, 13.4\% (34/254) were infected with $T$. congolense savannah type, 3.5\% (9/254) with $T$. congolense forest type, $0.4 \%(1 / 254)$ with $T$. vivax and 5.1\% (13/254) with Trypanozoon; 4.3\% (11/254) with T. b. gambiense and $3.14 \%(8 / 254)$ with T. b. brucei. 
Table 1 Results of the entomological survey and the identification of blood meals as well as different species of trypanosomes according to each trap

\begin{tabular}{|c|c|c|c|c|c|c|c|c|c|c|c|c|}
\hline Trap number & Latitude & Longitude & Flies Trapped & Dissected flies & ADP & Blood meals & Teneral & TB & TBG & TV & TCS & TCF \\
\hline \multicolumn{13}{|c|}{ Site A (Malanga village) } \\
\hline A1 & $5.54660^{\circ}$ & $14.34885^{\circ}$ & 23 & 12 & 5.75 & - & 3 & - & - & - & - & 1 \\
\hline A2 & $5.54633^{\circ}$ & $14.34854^{\circ}$ & 14 & 3 & 3.5 & - & 1 & - & - & - & - & - \\
\hline A3 & $5.54692^{\circ}$ & $14.34871^{\circ}$ & - & - & - & - & - & - & - & - & - & - \\
\hline A4 & $5.54774^{\circ}$ & $14.34972^{\circ}$ & 5 & - & 1.25 & - & - & - & - & - & - & - \\
\hline A5 & $5.54687^{\circ}$ & $14.35132^{\circ}$ & 32 & 6 & 8 & - & 1 & - & - & - & - & - \\
\hline A6 & $5.54742^{\circ}$ & $14.35162^{\circ}$ & 96 & 17 & 24 & $2 \mathrm{HB}, 2 \mathrm{UN}$ & 2 & - & - & - & 2 & - \\
\hline A7 & $5.54710^{\circ}$ & $14.35212^{\circ}$ & 33 & 5 & 8.25 & - & - & - & - & - & - & - \\
\hline A8 & $5.54658^{\circ}$ & $14.35253^{\circ}$ & 35 & 10 & 8.75 & $2 P B$ & 3 & - & - & - & 2 & - \\
\hline A9 & $5.54418^{\circ}$ & $14.35217^{\circ}$ & 71 & 9 & 17.75 & $2 \mathrm{~PB}, 1 \mathrm{HB}$ & 4 & 1 & 1 & - & - & 1 \\
\hline A10 & $5.54348^{\circ}$ & $14.35270^{\circ}$ & 47 & 11 & 11.75 & $4 \mathrm{~PB}, 1 \mathrm{HB}$ & 1 & 1 & 1 & - & 3 & 2 \\
\hline A11 & $5.54320^{\circ}$ & $14.35263^{\circ}$ & 18 & 3 & 4.5 & $1 \mathrm{~PB}$ & - & - & - & - & 1 & - \\
\hline A12 & $5.54494^{\circ}$ & $14.35270^{\circ}$ & 28 & 9 & 7 & 3 PB & 2 & - & - & - & 3 & - \\
\hline A13 & $5.54412^{\circ}$ & $14.35347^{\circ}$ & 5 & 3 & 1.25 & - & - & 1 & 1 & - & - & - \\
\hline A14 & $5.54497^{\circ}$ & $14.35431^{\circ}$ & - & - & - & - & - & - & - & - & - & - \\
\hline A15 & $5.54694^{\circ}$ & $14.35401^{\circ}$ & 51 & 10 & 12.75 & - & 3 & - & - & - & 1 & - \\
\hline Total & & & 458 & 98 & 7.3 & $12 \mathrm{~PB}, 4 \mathrm{HB}, 2 \mathrm{UN}$ & 20 & 3 & 3 & 0 & 12 & 4 \\
\hline \multicolumn{13}{|c|}{ Site B (Malanga gare) } \\
\hline B1 & $5.57142^{\circ}$ & $14.35199^{\circ}$ & 7 & 6 & 1.75 & $1 \mathrm{UN}$ & 2 & 1 & 1 & - & - & - \\
\hline B2 & $5.57081^{\circ}$ & $14.35233^{\circ}$ & 9 & 2 & 2.25 & $1 \mathrm{~PB}$ & - & - & - & - & - & - \\
\hline B3 & $5.57023^{\circ}$ & $14.35267^{\circ}$ & 25 & 15 & 6.25 & $3 \mathrm{HB}$ & 1 & - & - & 1 & 1 & 1 \\
\hline B4 & $5.56977^{\circ}$ & $14.35247^{\circ}$ & 18 & 9 & 4.5 & $2 P B$ & - & 1 & 1 & - & - & 1 \\
\hline B5 & $5.56973^{\circ}$ & $14.35330^{\circ}$ & 6 & 7 & 1.5 & $1 \mathrm{HB}$ & - & 1 & 1 & - & 1 & - \\
\hline B6 & $5.56927^{\circ}$ & $14.35414^{\circ}$ & 13 & 7 & 3.25 & $3 \mathrm{~PB}$ & - & - & - & - & 1 & - \\
\hline B7 & $5.56967^{\circ}$ & $14.35420^{\circ}$ & 59 & 17 & 14.75 & $6 \mathrm{HB}$ & 1 & 3 & 3 & - & 3 & 1 \\
\hline B8 & $5.56944^{\circ}$ & $14.35452^{\circ}$ & 75 & 22 & 18.75 & $4 \mathrm{~PB}, 1 \mathrm{HB}$ & 3 & - & - & - & 2 & 1 \\
\hline B9 & $5.56864^{\circ}$ & $14.35313^{\circ}$ & 40 & 3 & 10 & $3 \mathrm{HB}$ & 1 & 1 & 1 & - & - & - \\
\hline B10 & $5.56828^{\circ}$ & $14.35343^{\circ}$ & 24 & 8 & 6 & - & 2 & - & - & - & - & - \\
\hline B11 & $5.56776^{\circ}$ & $14.35339^{\circ}$ & 26 & 15 & 6.5 & - & 1 & 1 & 1 & - & 1 & - \\
\hline B12 & $5.56758^{\circ}$ & $14.35390^{\circ}$ & 23 & 17 & 5.75 & $2 P B$ & 3 & - & - & - & 1 & - \\
\hline B13 & $5.56748^{\circ}$ & $14.35422^{\circ}$ & 7 & 3 & 1.75 & - & 1 & - & - & - & - & - \\
\hline B14 & $5.56768^{\circ}$ & $14.35495^{\circ}$ & 21 & 15 & 5.25 & $2 \mathrm{~PB}$ & 4 & - & - & - & 2 & - \\
\hline B15 & $5.56720^{\circ}$ & $14.35477^{\circ}$ & 59 & 12 & 14.75 & $2 U N$ & 2 & 1 & - & - & 2 & - \\
\hline B16 & $5.56635^{\circ}$ & $14.35462^{\circ}$ & 21 & 9 & 5.25 & $3 \mathrm{~PB}$ & 1 & - & - & - & - & - \\
\hline B17 & $5.56583^{\circ}$ & $14.35449^{\circ}$ & 9 & 2 & 2.25 & - & - & 1 & - & - & 1 & - \\
\hline B18 & $5.56777^{\circ}$ & $14.35561^{\circ}$ & 49 & 30 & 12.25 & $6 \mathrm{~PB}, 2 \mathrm{HB}$ & 1 & - & - & - & 7 & 1 \\
\hline Total & & & 491 & 198 & 6.82 & $23 \mathrm{~PB}, 16 \mathrm{HB}, 3 \mathrm{UN}$ & 22 & 10 & 8 & 1 & 22 & 5 \\
\hline
\end{tabular}

$H B$ : Blood meals taken on humans; PG: Blood meals taken on pigs; UN: blood meals not identified despite the amplification of cytochrome B gene; ADP: apparent density of tsetse flies per trap per day; TB: Trypanosoma brucei s.l.; TBG: Trypanosoma brucei gambiense; TCF: Trypanosoma congolense forest type; TCS: Trypanosoma congolense savannah type; TV: Trypanosoma vivax.

\section{Discussion}

To improve our knowledge on the epidemiological status of HAT and AAT in the Malanga sleeping sickness focus, tsetse flies were trapped and the blood meal origins, as well as different species of trypanosomes, were identified in tsetse mid-guts. Glossina palpalis palpalis was the only tsetse fly species trapped in this focus, meaning that it is the main vector of the disease in this 
area. The high percentage of dead flies could be due to the high temperature observed during the dry season. In addition, the higher ADP may be another explanation. For instance, the average ADP observed during this study was about 7; indicating that more than 231 tsetse flies were trapped per day. This considerable number of flies trapped per day was difficult to dissect on the same day and some flies died overnight despite the fact that the tubes containing these flies were wrapped up in wet floor cloth.

The percentage (6.33\%) of residual blood meals obtained here is higher than the values obtained $(2.8 \%$ and 4.7\%; [20] and [16], respectively) for the same tsetse species in southern Cameroon. The difference can be explained by the composition of the fauna as well as the number of domestic and wild animal species found in each focus. In the forest HAT focus of southern Cameroon where different wild animal species are found $[19,21]$, very few domestic animals (especially pigs) are bred. Therefore, tsetse flies may have difficulty taking blood meals due to the vivacity of wild animals compared to pigs and other domestic animals. In the Malanga focus where considerable numbers of pigs are found, wild animals are rare [22]. Tsetse flies could easily take blood meals on domestic animals like pigs. This hypothesis is strengthened by the high percentage (58.3\%) of pig blood meals found in tsetse flies from this focus. In the Malanga HAT focus, humans appeared as the second source of blood meals for tsetse flies, since about $33 \%$ of the blood meals were taken on humans; illustrating thus an important human/tsetse contact in this focus. Moreover, the identification of T. b. gambiense in the mid-gut of tsetse flies of this focus strengthens these human/tsetse contacts and suggests an active transmission HAT in the Malanga focus. This also suggests a transmission cycle including human and tsetse flies.

The results of the identification of different species of trypanosomes seem to indicate that the species-specific PCR method is more sensitive than the ITS, since 13 Trypanozoon infections were identified by specific PCR, whereas, only 4 of these infections were identified by ITS. These results are in line with those obtained by Desquenes et al. [14] who showed that a specific PCR method was more sensitive than the ITS in identifying different trypanosome species in African livestock. Using the ITS, different trypanosome species can be revealed with only two PCR rounds, because each trypanosome species will generate a DNA fragment with a specific molecular weight. As multiple infections are common in tsetse flies and mammals, this technique appears, therefore, very useful in large scale studies aiming to identify natural trypanosome infections. Despite the low sensitivity of ITS, this technique enables the identification of several trypanosomes species; allowing thus a reduction of the analysis costs. With this technique, a considerable number of samples can be analyzed in a reasonable time and different trypanosome infections can be revealed.

The low infection rate of T. brucei s.l. in tsetse flies of the Malanga focus is in line with results obtained by Makumyaviri et al. [22] who identified very few infections of this trypanosome in domestic animals of the same area more than twenty years ago. Eleven of the 13 T. brucei infections were due to T. b. gambiense, suggesting that most of the T. brucei s.l. infections found in this area were due to T. b. gambiense. These results indicate a current circulation of T. b. gambiense in the HAT focus of Malanga. Our results suggest very low transmission of $T . b$. brucei in this focus because only 8 tsetse flies were found with this infection. These results are in line with those obtained in animals where only one domestic animal was found infected with this parasite [22]. The rarity of $T . b$. brucei in this zone can probably be explained by the fact that wild animals, known as being the reservoir of these parasites as well as the source of new infections, are scarce in this focus [22].

The identification of mixed infections involving different trypanosome species confirms previous results [6,2327], and reflects either the prevalence of such infections in vertebrate hosts of this area, or frequent feeding of tsetse flies on animals infected with different trypanosome species. However, the low percentage (7.02\%) of mixed infections involving different trypanosome species does not corroborate results obtained in other African regions where a high prevalence of mixed infections has been reported in tsetse flies [6,23-28]. The discrepancy between the percentages of mixed infections involving different trypanosome species can be explained by the availability of a suitable host for each trypanosome species. Despite the fact that the factors determining the distribution and abundance of trypanosomes are poorly known, it is likely, that most of these factors include the availability of suitable mammalian hosts [23]. The presence of mixed infections of T. b. brucei and T. b. gambiense confirms results obtained in Cameroon where about $69 \%$ of T. b. gambiense mid-gut infections were mixed with T. b. brucei [29].

Among the trypanosomes of the subgenus Nannomonas, T. congolense was the most common species found, and $T$. congolense savannah type the most prevalent. These results are in line with those reported in Southern Africa [23]. The high infection rate of trypanosomes belonging to Nannomonas, especially $T$. congolense, corroborates results obtained in tsetse flies of West, Central [25] and East Africa [23]. This is in line with the results obtained in domestic animals of the same area [22]. The high prevalence of $T$. congolense savannah type can be explained by geographical localization of the study area, which is located in the savannah zone. 
The identification of different species of trypanosomes in tsetse of the Malanga HAT focus indicates the presence of trypanosomes causing HAT and AAT. Although the presence of $T . b$. gambiense in tsetse mid-guts is not proof of mature infection, since some mid-gut infections do not develop to maturation in the salivary glands, the identification of this parasite as well as the human blood meals in tsetse mid-guts indicates the circulation of $T . b$. gambiense between tsetse flies and man. This suggests an active transmission of HAT in the Malanga focus. Our results suggest that a flare up of the disease can occur at any time if control strategies are not put in place. This is of crucial importance for the HAT control program, especially at this moment in time, where active case detection is scarce in foci presenting low disease prevalence. The considerable number of pig blood meals associated with the identification of $T . b$. gambiense in tsetse mid-guts suggests that investigations on the animal reservoir of HAT at Malanga should be carried out.

\section{Conclusion}

The results of this study (blood meal origins and different trypanosome species) have provided data that may help in the control and the surveillance of HAT and AAT. They may help to localize areas with higher transmission risk where vector control could be focused. If such control is undertaken, a direct effect will be observed on the animal reservoirs as well as on the number of infected flies. These approaches may be useful, especially currently, where the elimination of the disease has been envisaged in most HAT foci.

\section{Competing interests}

The authors declare that they have no competing interests.

\section{Authors' contributions}

GS participated to the conception, the design of the study, the tsetse fly sampling and the drafting of the manuscript. BS was involved in the identification of blood meals as well as different species of trypanosomes. FN was involved in the conception of the study and the drafting of the manuscript. PL participated to the conception and the tsetse fly sampling. PM participated to the tsetse fly sampling. JM participated to the tsetse fly sampling. EZ participated to the tsetse fly sampling. RDD participated to the design of the study, the conception of pictures and the drafting of the manuscript. TA was involved in the conception of the study and the drafting of the manuscript. All authors read and approved the final version of the manuscript.

\section{Acknowledgements}

This work was supported by "European Foundation Initiative for NTDs" (EFINTD), the University of Yaoundé I and the University of Dschang.

\section{Author details}

${ }^{1}$ Department of Biochemistry, Faculty of Science, University of Dschang, PO Box 67, Dschang, Cameroon. 'Faculty of Science, University of Yaoundé 1, Yaoundé , Cameroon. ${ }^{3}$ Department of Tropical Medicine, School of Medicine, Kinshasa University, Kinshasa, Democratic Republic of Congo. ${ }^{4}$ Programme National de Lutte contre la Trypanosomiase Humaine Africaine, Kinshasa, Democratic Republic of the Congo. ${ }^{5}$ Institut National de Recherche Biomédicale, Kinshasa, Democratic Republic of the Congo. ${ }^{6}$ Department of Animal Health, Institute of Tropical Medicine, Nationalestraat 155, 2000
Antwerp, Belgium. ${ }^{7}$ Faculty of Medicine and Biomedical Sciences, University of Yaoundé 1, Yaounde, Cameroon.

Received: 13 August 2012 Accepted: 11 September 2012

Published: 19 September 2012

\section{References}

1. Simarro PP, Jannin J, Cattand P: The Human African Trypanosomiasis control and surveillance programme of the World Health Organization: The way forward. PLoS Negl Trop Dis 2011, 5:e1007.

2. Penchenier $L$, Wang $S$, Bureau P: Historique et évolution de la maladie du sommeil dans les pays de l'OCEAC. In La trypanosomiase dans les états de I'OCEAC: historique et actualité. Bul Liais Doc OCEAC 1996, 29:11-22.

3. Simarro PP, Jannin J, Cattang P: Eliminating Human African Trypanosomiasis: where do we stand and what comes next? Plos Med 2008, 5:55.

4. Njiokou F, Simo G, Mbida Mbida A, Truc P, Cuny G, Herder S: A study of host preference in tsetse flies using a modified heteroduplex PCR-based method. Acta Trop 2004, 91:117-120.

5. Boakye DA, Tang J, Truc P, Merriweather A, Unnasch TR: Identification of bloodmeals in haematophagous Diptera by cytochrome B heteroduplex analysis. Med Vet Entomol 1999, 13:282-287.

6. Majiwa PAO, Otieno LH: Recombinant DNA probes reveal simultaneous infections of tsetse flies with different species. Mol Biochem Parasitol 1990, 40:245-254.

7. Majiwa PAO, Thatti E, Moloo SK, Nyeko JHP, Otieno LH, Maloo S: Detection of trypanosome infections in the saliva of tsetse flies and buffy-coat samples from antigenaemic but aparasitaemic cattle. Parasitology 1994, 108:312-322.

8. Moser DR, Cook GA, Ochs DE, Bailey CP, McKane MR, Donelson JE: Detection of Trypanosoma congolense and Trypanosoma brucei subspecies by DNA amplification using polymerase chain reaction. Parasitology 1989, 99:57-66.

9. Bromidge T, Gibson W, Hudson KM, Dukes P: Identification of Trypanosoma brucei gambiense by PCR amplification of variant surface glycoprotein genes. Acta Trop 1993, 53:107-119.

10. MacLeod A, Turner CMR, Tait A: A high level of mixed Trypanosoma brucei infections in tsetse flies detected by three hypervariable minisatellites. Mol Biochem Parasitol 1999, 102:237-248.

11. Biteau N, Bringaud F, Gibson W, Truc P, Baltz T: Characterization of Trypanozoon isolates using a repeated coding sequence and microsatellite markers. Mol Biochem Parasitol 2000, 105:185-201.

12. Radwanska M, Claes F, Magez S, Magnus E, Perez-Morga D, Pays E, Büscher $P$ : Novel primer sequences for polymerase chain reaction-based detection of Trypanosoma brucei gambiense. Am J Trop Med Hyg 2002, 67:289-295.

13. Gouteux J, Lancien J: Le piège pyramidal à tsé-tsé (Diptera: Glossinidae) pour la capture et la lutte. Essais comparatifs et description de nouveaux systèmes de capture. Trop Med Parasitol 1986, 37:61-66.

14. Desquesnes M, McLaughlin G, Zoungrana A, Davila AM: Detection and identification of Trypanosoma of African livestock through a single PCR based on internal transcribed spacer 1 of rDNA. Int J Parasitol 2001, 31:610-614.

15. Masiga DK, Smyth AJ, Hayes P, Bromidge TJ, Gibson WC: Sensitive detection of trypanosomes in tsetse flies by DNA amplification. Int J Parasitol 1992, 22:909-918.

16. Farikou O, Njiokou F, Simo G, Asonganyi T, Cuny G, Geiger A: Tsetse fly blood meal modification and trypanosome identification in two sleeping sickness foci in the forest of southern Cameroon. Acta Trop 2010, 116:81-88.

17. Simo G, Asonganyi T, Nkinin SW, Njiokou F, Herder S: High prevalence of Trypanosoma brucei gambiense group 1 in pigs from the Fontem sleeping sickness focus in Cameroon. Vet Parasitol 2006, 139:57-66.

18. Herder S, Simo G, Nkinin S, Njiokou F: Detection of trypanosomes in wild animals from southern Cameroon using polymerase chain reaction (PCR). Parasite 2002, 9:345-349.

19. Njiokou F, Simo G, Nkinin SW, Laveissière C, Herder S: Infection rate of Trypanosoma brucei s.l, T. vivax, T. congolense «forest type» and T. simiae in small wild vertebrate in south Cameroon. Acta Trop 2004, 92:139-146.

20. Simo G, Njiokou F, Mbida Mbida JA, Njitchouang GR, Herder S, Asonganyi T, Cuny G: Tsetse fly host preference from sleeping sickness foci in Cameroon: epidemiological implications. Infect Genet Evol 2008, 8:34-39. 
21. Njiokou F, Laveissière C, Simo G, Nkinin S, Grébaut P, Cuny G, Herder S: Wild fauna as a probable animal reservoir for Trypanosoma brucei gambiense in Cameroon. Infect Genet Evol 2006, 6:147-153.

22. Makumyaviri A, Mehlitz D, Kageruka P, Kazumba GL, Molisho D: Le réservoir animal de Trypanosoma brucei gambiense au Zaire: infections trypanosomiennes dans deux foyers du Bas-zaire. Trop Med Parasitol 1989, 40:258-262.

23. Nimpaye H, Njiokou F, Njine T, Njitchouang GR, Cuny G, Herder S, Asonganyi T, Simo G: Trypanosoma vivax, T. congolense "forest type" and T. simiae: prevalence in domestic animals of sleeping sickness foci of Cameroon. Parasite 2011, 18:9-171.

24. Masiga DK, McNamara JJ, Laveissière C, Truc P, Gibson WC: A high prevalence of mixed infections in tsetse flies in Sinfra, Côte d'Ivoire, detected by DNA amplification. Parasitology 1996, 112:75-80.

25. Morlais I, Grébaut P, Bodo JM, Djoha S, Cuny G, Herder S: Detection and identification of trypanosomes by polymerase chain reaction in wild tsetse flies in Cameroon. Acta Trop 1998, 70:109-117.

26. Lehane MJ, Msangi AR, Whitacker CJ, Lehane SM: Grouping of trypanosome species in mixed infections in Glossina pallidipes. Parasitology 2000, 120:583-592.

27. Jamonneau V, Ravel S, Koffi M, Kaba D, Zeze DG, Ndri L, Sane B, Coulibaly B, Cuny G, Solano P: Mixed infections of trypanosomes in tsetse and pigs and their epidemiological significance in a sleeping sickness focus of Côte d'Ivoire. Parasitology 2004, 129:693-702.

28. McNamara JJ, Laveissière C, Masiga DK: Multiple trypanosome infections in tsetse in Côte d'Ivoire detected by PCR analysis and DNA probes. Acta Trop 1995, 59:85-92.

29. Simo G, Njitchoua ng GR, Njiokou F, Cuny G, Asonganyi T: Trypanosoma brucei s.l.: Microsatellite markers revealed high level of multiple genotypes in the mid-guts of wild tsetse flies of the Fontem sleeping sickness focus of Cameroon. Exp Parasitol 2011, 128:272-8.

doi:10.1186/1756-3305-5-201

Cite this article as: Simo et al.: Identification of different trypanosome species in the mid-guts of tsetse flies of the Malanga (Kimpese)

sleeping sickness focus of the Democratic Republic of Congo. Parasites \& Vectors 2012 5:201.

\section{Submit your next manuscript to BioMed Central and take full advantage of:}

- Convenient online submission

- Thorough peer review

- No space constraints or color figure charges

- Immediate publication on acceptance

- Inclusion in PubMed, CAS, Scopus and Google Scholar

- Research which is freely available for redistribution 